\title{
Magnetohydrodynamic oscillatory flow of a physiological fluid in an irregular channel
}

\author{
B. Venkateswarlu ${ }^{1} \cdot$ K. Bhagya Lakshmi ${ }^{2} \cdot$ S. Samantha Kumari ${ }^{3} \cdot$ P. V. Satya Narayana ${ }^{4}(\mathbb{C}$
}

(C) Springer Nature Switzerland AG 2019

\begin{abstract}
The impact of heat source on magnetohydrodynamic oscillatory flow of a chemically reacting viscoelastic fluid in an asymmetric wavy channel is analysed. The governing flow equations are transformed into ODEs by utilizing proper nondimensional variables. The subsequent ordinary differential equations are solved analytically. The effects of different flow parameters on the fluid flow, thermal and species distributions as well as rate of heat and mass transfer coefficients are examined graphically. It is pointed out that the velocity of fluid is parabolic with extreme value along the channel centreline and minimum at the walls. The magnitude of fluid velocity increases with an increase in porous parameter and high heat transport of a system is due to the presence of oscillatory flow.
\end{abstract}

Keywords Visco-elastic fluid · Heat source $\cdot$ MHD · Oscillatory flow · Asymmetric channel

\section{List of symbols}

$a_{1}, b_{1} \quad$ Amplitudes of the wavy walls

$a, b \quad$ Amplitude ratios

$B_{0} \quad$ Electromagnetic induction

$C^{*} \quad$ Fluid concentration

$C_{1}, C_{2} \quad$ Concentrations at walls

$C_{p} \quad$ Specific heat at constant pressure

$d_{1}+d_{2} \quad$ Width of channel

$d \quad$ Mean half width of the channel

$D_{a} \quad$ Darcy number

g Gravitational force

Gc Modified Grashof number

$\mathrm{Gr} \quad$ Grashof number

$H_{0} \quad$ Intensity of magnetic field

$H_{1}, H_{2} \quad$ Inner and outer walls

$K \quad$ Porous medium shape factor

$\mathrm{Kr} \quad$ Chemical reaction parameter

$k^{*} \quad$ Porous permeability coefficient

$k \quad$ Thermal conductivity

M Hartmann number

$\mathrm{Nu} \quad$ Nusselt number

$\mathrm{Pe} \quad$ Peclet number $p \quad$ Pressure

$Q \quad$ Heat source parameter

$q \quad$ Radiative heat flux

Re Reynolds number

$R \quad$ Radiation parameter

Sc Schmidt number

Sh Sherwood number

$T \quad$ Fluid temperature

$T_{1}, T_{2} \quad$ Temperatures at walls

$t \quad$ Time

$U \quad$ Flow mean velocity

$u \quad$ Axial velocity

Greek symbols

$\theta \quad$ Fluid temperature

$\beta_{T} \quad$ Coefficient of thermal expansion

$\beta_{C} \quad$ Coefficient of mass expansion

$\mu \quad$ Coefficient of viscosity

$\mu_{e} \quad$ Magnetic permeability

$\sigma_{c} \quad$ Conductivity of the fluid

$\rho \quad$ Fluid density

$u \quad$ Kinematics viscosity coefficient

P. V. Satya Narayana, pvsatya8@yahoo.co.in | 'Department of Mathematics, Walchand Institute of Technology, Solapur, MH 413006, India. ${ }^{2}$ CMR Technical Campus, Kandlakoya, Hyderabad, Telangana 501401, India. ${ }^{3}$ Department of Mathematics, JNTUA, Anantapuramu, AP, India. ${ }^{4}$ Department of Mathematics, SAS, VIT, Vellore, TN 632 014, India. 
$\lambda \quad$ Wave length

$\omega \quad$ Frequency of the oscillation

a Radiation absorption coefficient

$\tau \quad$ Skin friction coefficient

\section{Introduction}

Recently, many researchers [1-10] explored the MHD nonNewtonian fluid flows over various geometrical models in view of their significance in many engineering and industrial applications such as food processing, making a paper, slurry transporting etc.,. Physiological fluid is one of the non-Newtonian viscoelastic fluids which play an important role in many transport phenomenon's (such as blood circulation, cancer treatment, transport of drugs, drug carriers). It is observed that the viscoelastic property of blood is less prominent with rising shear rate and gliding of cells (see Refs. [11, 12]). The blood flow properties are determined by the cell aggregation tendency, red cell concentration and mechanical relation between the particles (see Refs. [13-19]). The experimental studies reveal that, in case of cancer treatment by radiotherapy, chemotherapy or surgery, they destroy both malignant and normal cells. In view of these issues, Nikiforov [20] developed an electromagnetic hyperthermia technique where the dangerous cancer cells are exposed to a temperature field greater than $41^{\circ} \mathrm{C}$ with the help of thermal radiation. In this method a magnetic liquid is injected to the malignant tissues and then exposing the framework to an alternative current. Due to this, the temperature is produced in the infused magnetic fluid and there by the cancer cells get destroyed (see Ref. [21]). Raftari and Vajravelu [22] studied the impact of magnetic field on a visco-elastic fluid flow through a channel by applying HAM analysis. Singh [23, 24] investigated the influence of slip and thermal radiation on visco-elastic fluid flow past a vertical channel. Abdalla and Abo Dahab [25] explored the rotating and non-Newtonian fluid effects in an asymmetric channel with peristaltic transport. Many researchers [26-29] considered the different mathematical models to study the impact of viscoelastic fluid flow through porous channels.

Now a days chemical reactions play a significant role in many science and engineering applications at different stages such as; chemical engineering processes, moisture over agricultural fields, energy transfer in a cooling tower power, cooling industry for drying, and the flow in desert cooler etc.,. In view of these, the visco-elastic fluid flow through a porous channel with chemical reaction was estimated analytically by Devika et al. [30]. Gireesha and Mahanthesh [31] explored the chemically reacting heat transfer flow of viscoelastic fluid through non uniform channels. Nayak et al. [32] analyzed the first order chemical reaction effects on viscoelastic fluid in the presence of porous medium. Venkateswarlu et al. [33] contemplated the chemically reacting viscoelastic fluid flow past a vertical channel.

It is also worth noting that most of afore mentioned studies have been considered in channels. Therefore, it would be interesting to explore the study of non-Newtonian fluid in an asymmetric wavy channel. In this way, the main aim of the present work is to investigate the visco-elastic fluid behavior of MHD oscillatory flow in an irregular channel. The governing equations are solved analytically. Impact of various flow parameters on the fluid flow characteristics are analyzed through graphs. Further, the comparison of the skin friction coefficient and Nusselt number for various values of magnetic field and heat source parameters are displayed in Table 1.

\section{Mathematical formulation}

Unsteady incompressible, chemically reacting and thermally radiating non-Newtonian fluid is considered in an irregular channel with heat source. A constant magnetic field of strength $B_{0}$ is assumed normal to the flow. The fluid flow along $x$-direction is generated due to the oscillatory pressure gradient at the walls as shown in Fig. 1. $T_{1}$ and $T_{2}$ are respectively the temperatures of the fluid at walls of the channel. The magnetic Reynolds number is assumed to be very small compared to the external one and hence the induced magnetic field can be negligible. The inner and outer walls $H_{1}$ and $H_{2}$ are respectively given by $[2,34]$

$H_{1}=d_{1}+a_{1} \cos \frac{2 \pi x}{\lambda}$ and $H_{2}=-d_{2}-b_{1} \cos \left(\frac{2 \pi x}{\lambda}+\phi\right)$,

where $a_{1}, b_{1}, d_{1}, d_{2}$ and $\phi$ satisfies the condition $a_{1}^{2}+b_{1}^{2}+2 a_{1} b_{1} \cos \phi \leq\left(d_{1}+d_{2}\right)^{2}, 0 \leq \varphi \leq \pi$.

The governing flow, energy and concentration equations are given by $[2,26,34]$

Table 1 Comparison of skin friction coefficient and Nusselt number for different values of $M$ and $Q$ respectively

\begin{tabular}{llllll}
\hline M & $\tau$ & & $\mathrm{Q}$ & $\mathrm{Nu}$ & \\
\cline { 2 - 3 } & Ref. [2] & Present & & Ref. [2] & Present \\
\hline 1.0 & 3.2578 & 3.257683 & 0.1 & 1.4152 & 1.415587 \\
2.0 & 3.2847 & 3.284695 & 0.3 & 2.0280 & 2.028342 \\
3.0 & 3.3297 & 3.322859 & 0.5 & 3.1336 & 3.133548 \\
4.0 & 3.3928 & 3.392673 & 0.7 & 5.8087 & 5.808621 \\
\hline
\end{tabular}




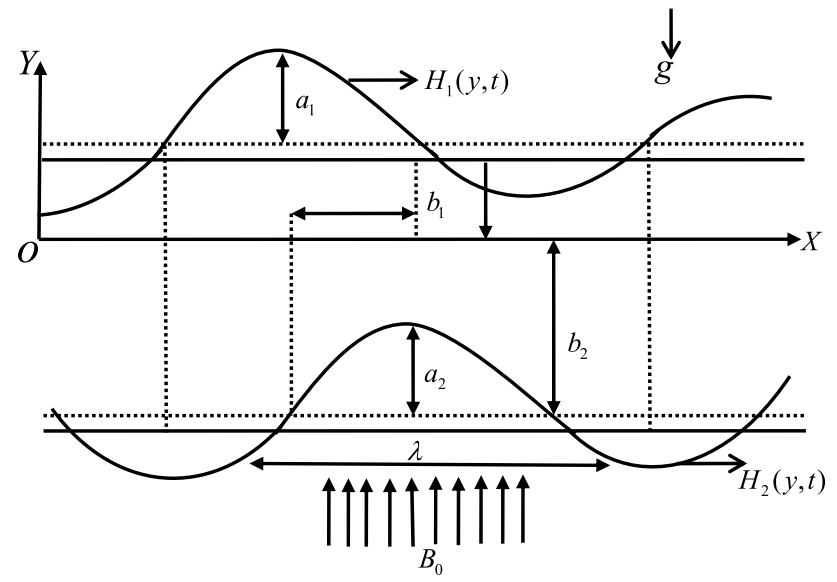

Fig. 1 Physical model of the problem

$$
\begin{aligned}
\frac{\partial U^{*}}{\partial t}= & -\frac{1}{\rho} \frac{\partial p}{\partial x}+v \frac{\partial^{2} U^{*}}{\partial y^{2}}+g \beta_{T}\left(T^{*}-T_{2}\right) \\
& +g \beta_{C}\left(C^{*}-C_{2}\right)-\frac{\sigma B_{0}^{2} U^{*}}{\rho}-\frac{v}{k^{*}} U^{*}+\beta_{0} \frac{\partial^{3} U^{*}}{\partial y^{2} \partial t},
\end{aligned}
$$

$\frac{\partial T}{\partial t}^{*}=\frac{k}{\rho c_{p}} \frac{\partial^{2} T^{*}}{\partial y^{2}}-\frac{1}{\rho c_{p}} \frac{\partial q}{\partial y}+\frac{Q_{H}}{\rho c_{p}}\left(T^{*}-T_{2}\right)$,

$\frac{\partial C^{*}}{\partial t}=D \frac{\partial^{2} C^{*}}{\partial y^{2}}-K_{r}^{*}\left(C^{*}-C_{2}\right)$,

The boundary conditions are defined as $[2,34]$

$U^{*}=0, \quad T^{*}=T_{1}, \quad C^{*}=C_{1}, \quad$ on $\quad y=H_{1}$,

$U^{*}=0, \quad T^{*}=T_{2}, \quad C^{*}=C_{2}$, on $y=H_{2}$,

The thermal radiative is defined by Ogulu and Bestman [35] as

$\frac{\partial q}{\partial y}=4 \alpha^{2}\left(T_{2}-T^{*}\right)$

where $\alpha^{2}=\int_{0}^{\infty} K_{\lambda w} \frac{\partial e_{b \lambda}}{\partial T} d \lambda$.The dimensionless variables are:

$\bar{x}=\frac{x}{\lambda}, \quad \bar{y}=\frac{y}{d_{1}}, \quad \bar{u}=\frac{U^{*}}{U}, \quad \bar{t}=\frac{t U}{d_{1}}, \quad h_{1}=\frac{H_{1}}{d_{1}}, \quad h_{2}=\frac{H_{2}}{d_{1}}, \quad d=\frac{d_{2}}{d_{1}}$,

$a=\frac{a_{1}}{d_{1}}, \quad b=\frac{b_{1}}{d_{1}}, \quad D_{a}=\frac{k^{*}}{d_{1}^{2}}, \quad \operatorname{Re}=\frac{U d_{1}}{v}, \quad Q=\frac{Q_{H} d_{1}^{2}}{k}, \quad K=\frac{d_{1}^{2}}{k^{*}}, \quad S c=\frac{D}{U d_{1}}$,

$K r=\frac{d_{1} K_{r}^{*}}{U}, \quad \bar{p}=\frac{d_{1}^{2} p}{\rho \nu \lambda U}, \quad P e=\frac{U d_{1} \rho c_{p}}{k}, \quad R=\frac{4 \alpha^{2} d_{1}^{2}}{k}, \quad M=\frac{\sigma B_{0}^{2} d_{1}^{2}}{\rho \nu}, \quad \beta=\frac{B_{0} U}{v d_{1}}$,

$G r=\frac{g \beta_{T}\left(T_{1}-T_{2}\right) d_{1}^{2}}{\nu U}, \quad G c=\frac{g \beta_{C}\left(C_{1}-C_{2}\right) d_{1}^{2}}{\nu U}, \quad \theta=\frac{T^{*}-T_{2}}{T_{1}-T_{2}}, \quad \phi=\frac{C^{*}-C_{2}}{C_{1}-C_{2}}$,
The corresponding boundary conditions are

$h_{1}=1+a \cos 2 \pi x$, and $h_{2}=-d-b \cos (2 \pi x+\phi)$,

where $a, b, d$ and $\phi$ satisfies the form $a^{2}+b^{2}+2 a b \cos \phi \leq(1+d)^{2}$.

In view of non-dimensional quantities Eqs. (2)-(4) can be written as (neglecting bar symbols)

$\operatorname{Re} \frac{\partial u}{\partial t}=-\frac{\partial p}{\partial x}+\frac{\partial^{2} u}{\partial y^{2}}+G r \theta+G c \phi-(M+1 / K) u+\beta \frac{\partial^{3} u}{\partial y^{2} \partial t}$

$P e \frac{\partial \theta}{\partial t}=\frac{\partial^{2} \theta}{\partial y^{2}}+(R+Q) \theta$

$\frac{\partial \phi}{\partial t}=S c \frac{\partial^{2} \phi}{\partial y^{2}}-K r \phi$

The corresponding non-dimensional boundary conditions are

$u=0, \quad \theta=1, \quad \phi=1, \quad$ on $\quad y=h_{1}$,

$u=0, \quad \theta=0, \quad \phi=0, \quad$ on $\quad y=h_{2}$,

\section{Method of solution}

In order to solve the system of PDEs (9)-(11) the following pressure gradient for purely oscillatory flow, velocity, thermal and concentrations fields are assumed as (see Refs. [34, 36-39])

$-\frac{\partial p}{\partial x}=\lambda e^{i \omega t}, \quad$ and $\quad f(y, t)=f_{0}(y) e^{i \omega t}$,

where $f$ is the $u, \theta$ and $\varphi, f_{0}$ is the $u_{0}, \theta_{0}$ and $\varphi_{0}$.

In view of Eq. (13), the Eqs. (9)-(11), are reduced to

$(1+i \omega \beta) \frac{d^{2} u_{0}}{d y^{2}}-n^{2} u_{0}=-\lambda-G r \theta_{0}-G c \phi_{0}$

$\frac{d^{2} \theta_{0}}{d y^{2}}+m^{2} \theta_{0}=0$ 
$\frac{d^{2} \phi_{0}}{d y^{2}}-l^{2} \phi_{0}=0$

The boundary conditions are

$u_{0}=0, \quad \theta_{0}=1, \quad \phi_{0}=1, \quad$ on $\quad y=h_{1}$,

$u_{0}=0, \quad \theta_{0}=0, \quad \phi_{0}=0, \quad$ on $\quad y=h_{2}$,

Solving the Eqs. (14)-(16) with the help of boundary conditions Eq. (17), we get the solutions for velocity, temperature and concentration are as follows

\section{Results and discussion}

The aim of this paper is to analyze the non-Newtonian fluid behavior and oscillatory flow of visco-elastic fluid in an irregular channel. The solutions of the Eqs. (18)-(20) are obtained by using MATLAB code and the results are displayed through Figs. 2, 3, 4, 5, 6, 7, 8, 9, 10, 11, 12, 13, 14 and 15 for various parameters entering into the problem. Table 1 displays the comparative study of skin friction and rate of heat transfer coefficients for Newtonian fluid with those of Ref. [2] by choosing $\omega=0.1, \lambda=0.1, t=0.1, \pi=3.14$,

$$
\begin{aligned}
u(y, t)= & \frac{\lambda}{n^{2}}(1+i \omega \beta) e^{i \omega t}+(1+i \omega \beta)\left\{\frac{\lambda}{n^{2}}+\frac{G r}{m^{2}+n^{2}}-\frac{G c}{l^{2}-n^{2}}\right\} \frac{\sinh n\left(h_{2}-y\right)}{\sinh n\left(h_{1}-h_{2}\right)} e^{i \omega t} \\
& +(1+i \omega \beta)\left\{\frac{\lambda}{n^{2}} \frac{\sinh n\left(y-h_{1}\right)}{\sinh n\left(h_{1}-h_{2}\right)}+\frac{G r}{m^{2}+n^{2}} \frac{\sin m\left(y-h_{2}\right)}{\sin m\left(h_{1}-h_{2}\right)}-\frac{G c}{l^{2}-n^{2}} \frac{\sinh /\left(y-h_{2}\right)}{\sinh /\left(h_{1}-h_{2}\right)}\right\} e^{i \omega t},
\end{aligned}
$$

$\theta(y, t)=\frac{\sin m\left(y-h_{2}\right)}{\sin m\left(h_{1}-h_{2}\right)} e^{i \omega t}$,

$\phi(y, t)=\frac{\sinh l\left(y-h_{2}\right)}{\sinh l\left(h_{1}-h_{2}\right)} e^{i \omega t}$

The skin friction coefficient, Nusselt and Sherwood number at the wall are define as follows $a=0.2, b=1.2, d_{1}=2.0$ and $\operatorname{Re}=0.1$. It is found to be good agreement between them.

Figure 2 displays the influence of porous parameter $K$ on the velocity profile for both Newtonian $(\beta=0)$ and non-Newtonian $(\beta \neq 0)$ cases. It is noticed that the velocity profiles attains the maximum value with increase of $K$. Physically, rising estimations of $K$ results the larger holes of porous medium and henceforth resistivity of the medium might be disregarded. Further, the velocity of Newtonian fluid is more than that of non-Newtonian fluid.

$$
\begin{aligned}
\tau= & \mu\left\{\frac{\partial u}{\partial y}\right\}_{y=h_{1}, h_{2}}=\mu(1+i \omega \beta)\left\{\frac{n G c}{12-n^{2}}-\frac{n G r}{m^{2}+n^{2}}-\frac{\lambda}{n}\right\} \frac{\cosh n\left(y-h_{2}\right)}{\sinh n\left(h_{1}-h_{2}\right)} e^{i \omega t} \\
& +\mu(1+i \omega \beta)\left\{\frac{\lambda \cosh n\left(y-h_{1}\right)}{n \sinh n\left(h_{1}-h_{2}\right)}-\frac{m G r}{m^{2}+n^{2}} \frac{\cosh m\left(y-h_{2}\right)}{\sinh m\left(h_{1}-h_{2}\right)}-\frac{I G c}{I^{2}-n^{2}} \frac{\cosh l\left(y-h_{2}\right)}{\sinh /\left(h_{1}-h_{2}\right)}\right\} e^{i \omega t},
\end{aligned}
$$

$N u=-\left(\frac{\partial \theta}{\partial y}\right)_{y=h_{1}, h_{2}}=-\frac{m \cos m\left(y-h_{2}\right)}{\sin m\left(h_{1}-h_{2}\right)} e^{i \omega t}=|J| \cos (\omega t+\psi)$,

$S h=-\left(\frac{\partial \phi}{\partial y}\right)_{y=h_{1}, h_{2}}=-\frac{I \cosh l\left(y-h_{2}\right)}{\sinh /\left(h_{1}-h_{2}\right)} e^{i \omega t}=|F| \cos (\omega t+\psi)$,

The amplitude and phase angle of the rate of heat and mass transfer are respectively given by $J=J_{r}+i J_{i}, \quad \psi=\tan ^{-1}\left(J_{i} / J_{r}\right), \quad F=F_{r}+i F_{i}, \quad \psi=\tan ^{-1}\left(F_{i} / F_{r}\right)$ where $R^{2}=K r+i \omega / S c, \quad m^{2}=Q+R-i \omega P e, \quad n^{2}=M+$ $1 / K+i \omega \operatorname{Re}$.
The impact of $M$ on $u$ is discussed in Fig. 3. It is noticed that the velocity along the boundary layer pulverizes with raising values of $M$. This is because of the way that the Lorentz force has the tendency to diminish the motion of the fluid and henceforth the fluid velocity diminishes.

The influence of $G r$ and $G c$ on $u$ is displayed in Fig. 4. It is observed that the velocity mounting with rising values of $G r$ and decline with $G c$ values. Physically, Gr signifies the general impact of the thermal buoyancy force to the viscous hydrodynamic force in the boundary layer. Thus, as $G r$ increases the velocity of the fluid reaches maximum 
Fig. 2 Influence of $\mathrm{K}$ on $u$

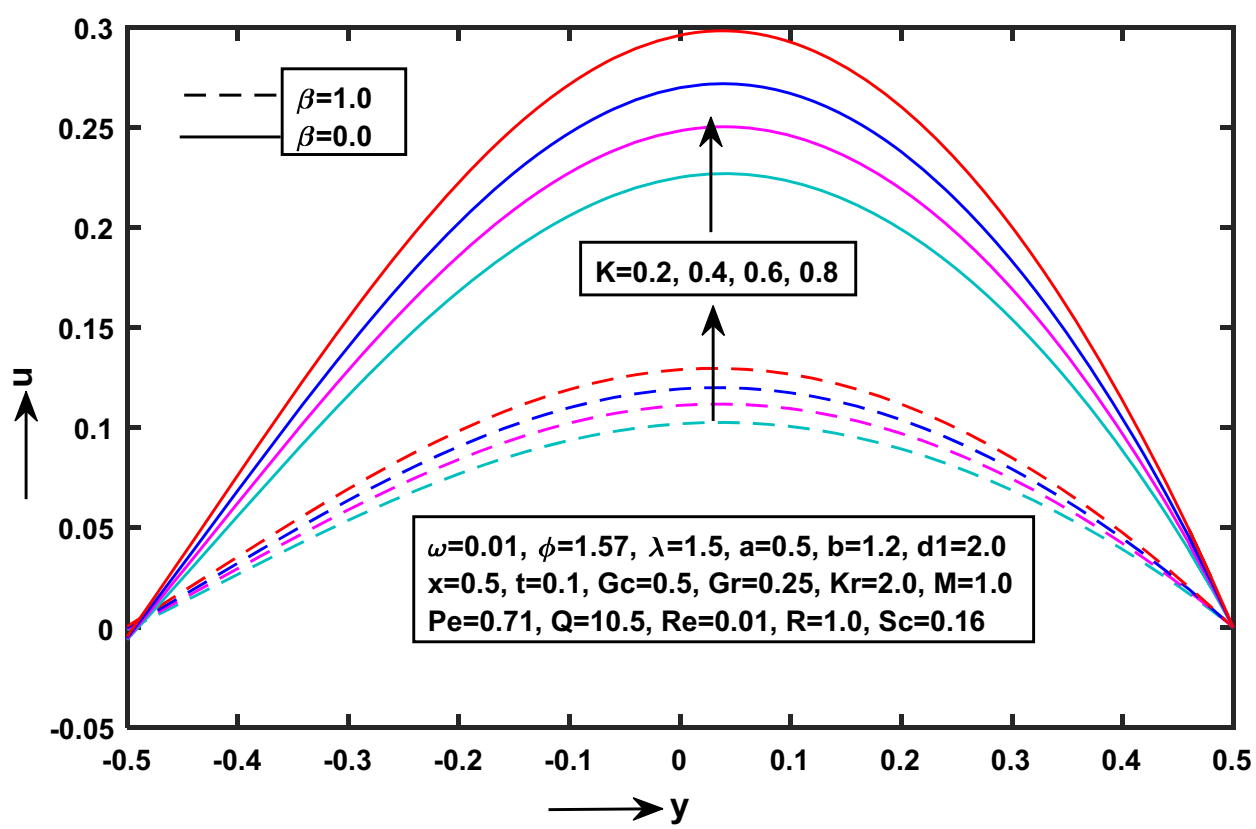

Fig. 3 Influence of $\mathrm{M}$ on $u$

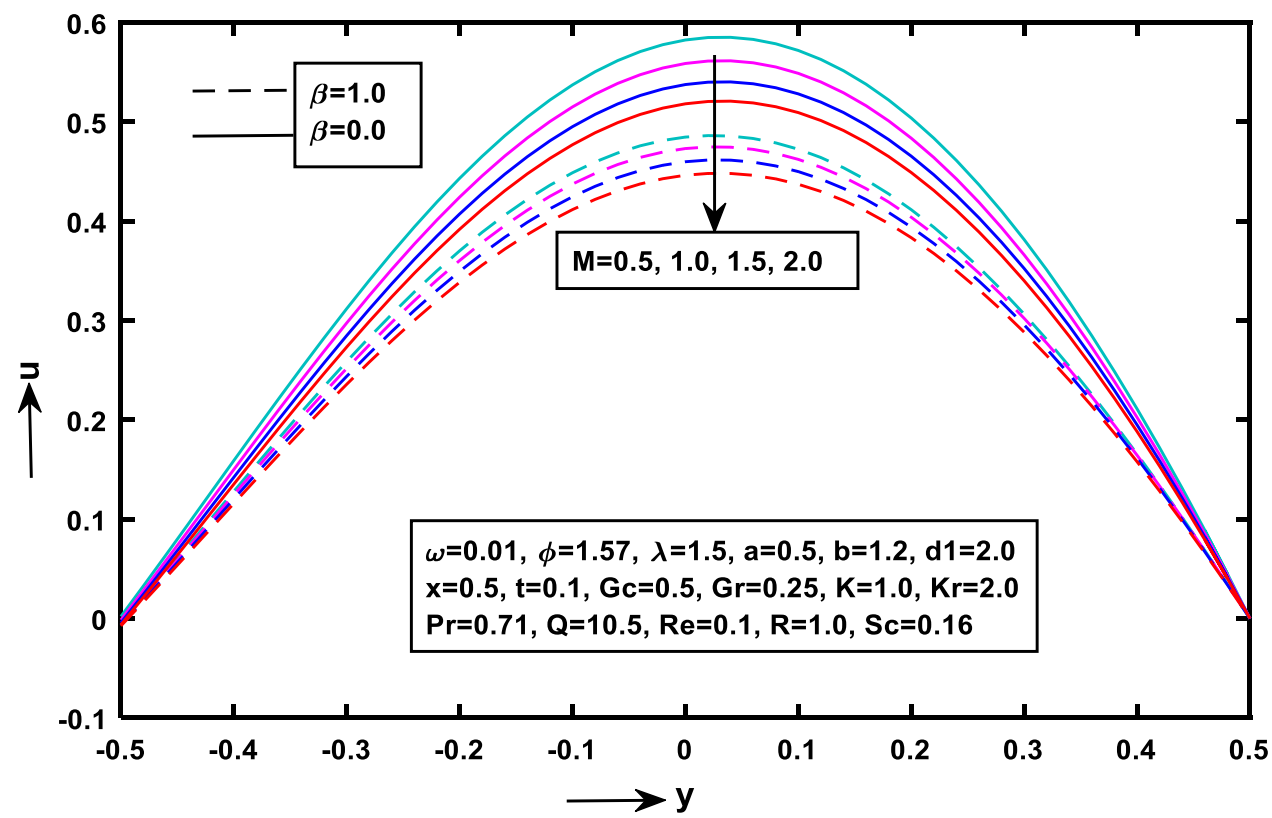

value in the middle region of the asymmetric channel and after that rots easily to the free stream velocity.

The influence of various values of $P e$ and Reynolds number Re on $u$ across the boundary layer is shown in Fig. 5. The Peclet number is the ratio of thermal energy convected to the fluid to the thermal energy conducted within the fluid and the Reynolds number is the ratio of inertial forces to viscous forces. The results of velocity profile increase with increase in $P e$ and reverse effect with 
Fig. 4 Influence of $G r$ and Gc on $u$

Fig. 5 Influence of $P e$ and $R e$ on $u$

rise of $R e$. These parameters may play an important role in convective heat transfer processes.

Figures 6 and 7 display the consequences of velocity and concentration of dissimilar values of $K r$ and $S C$ respectively. It is noticed that the velocity of the fluid increases and concentration decreases with rising values of chemical reaction parameter. In addition, Schmidt number shows reverse effect on these two profiles.
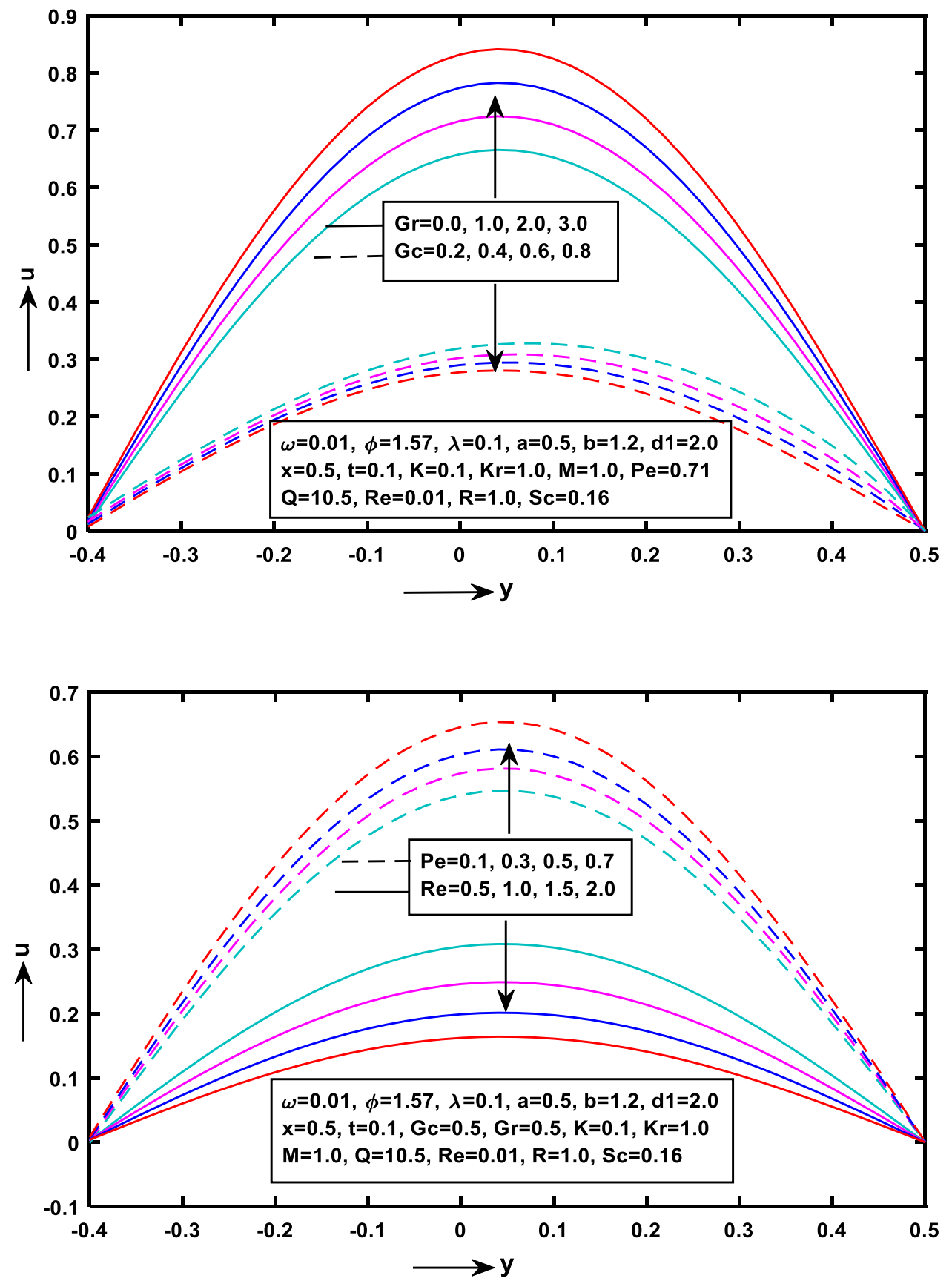

Physically, Schmidt number $\mathrm{Sc}$ is characterized as the ratio of momentum and mass diffusivity. Hence, as $S c$ increases the velocity and boundary layer thickness reduces and concentration of the fluid increases.

The influence of $R$ on $u$ is outlined in Fig. 8. It is seen that the velocity elevate with the ascent of $R$ in the flow regime. This is due to the fact that the fluid particles get the heat energy from the electromagnetic waves and 
Fig. 6 Influence of $K r$ and Sc on $u$

Fig. 7 Influence of $K r$ and Sc on $\phi$
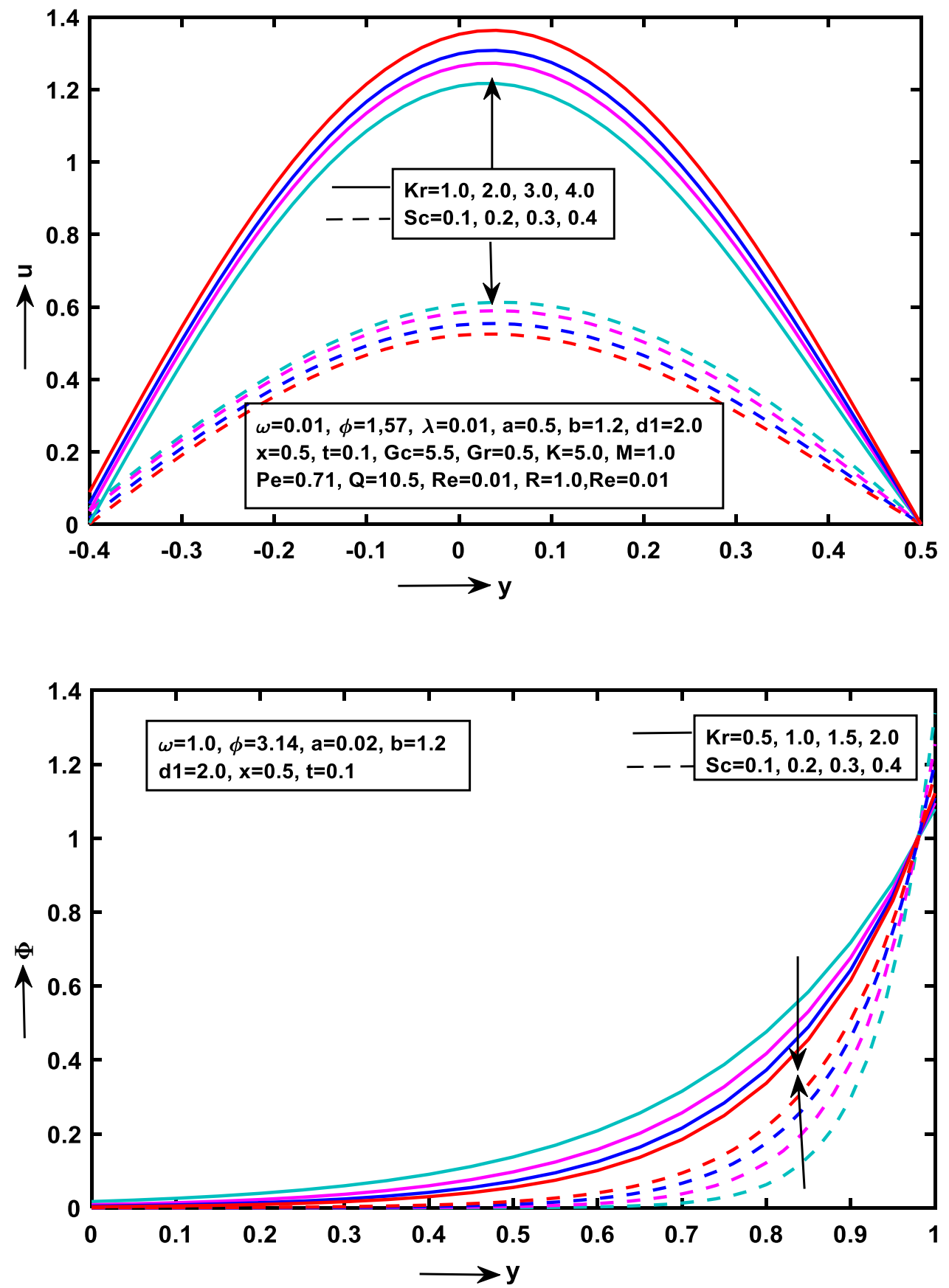

hence move very fast. Also the velocity of viscous fluid $(\beta=0)$ is more than that of non-Newtonian fluid $(\beta \neq 0)$.

Figure 9 represents the impact of $R, Q$ and $P e$ respectively on $\theta$. Plainly the temperature increment for every single developing value of $R, Q$ and $P e$ in the stream locale. Physically, the thermal radiation is responsible for energy transfer by the emission of electro-magnetic waves which carry energy away as the fluid flows along asymmetric wavy channels and consequently the thermal boundary layer thickness winds up more slender. Experimentally, when blood flow in capillaries, there is a significant increase in the thickness of boundary layer due to rise in thermal radiation. Hence, the temperature of the boundary layer is raised by an appreciable extent.

Figures 10 and 11 display $\tau$ for diverse values of $K$ and $M$ respectively, for both Newtonian and non-Newtonian 
Fig. 8 Influence of $R$ on $u$

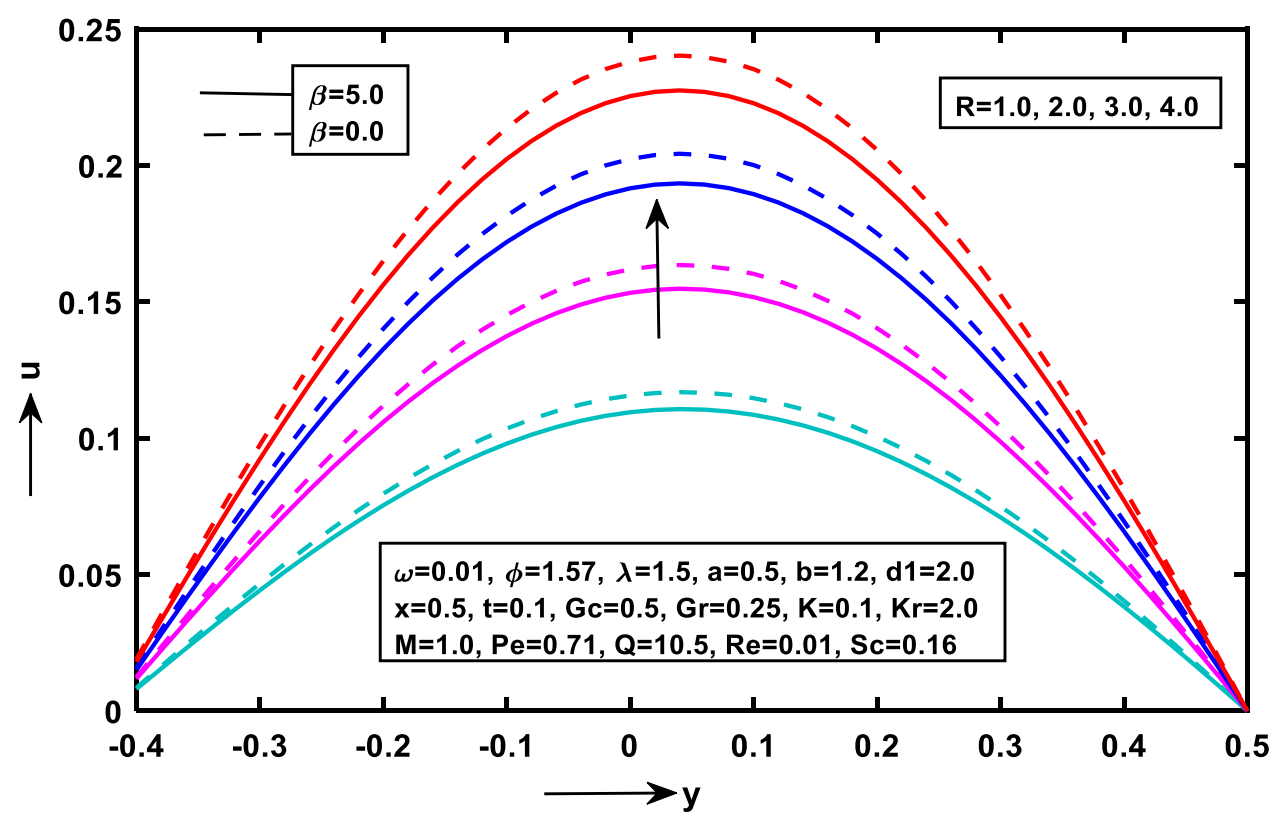

Fig. 9 Influence of $P e, Q$ and $R$ on $\theta$

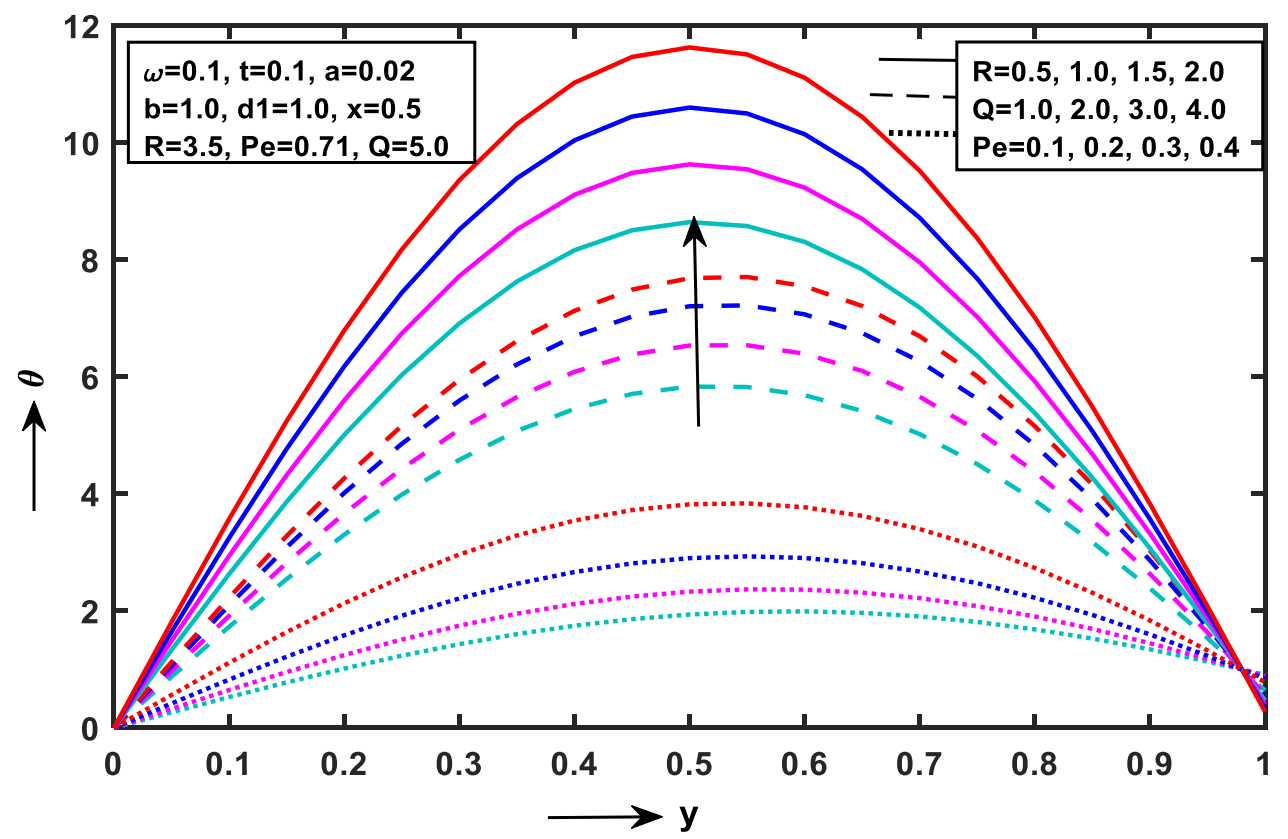


Fig. 10 Influence of $K$ on skin friction coefficient

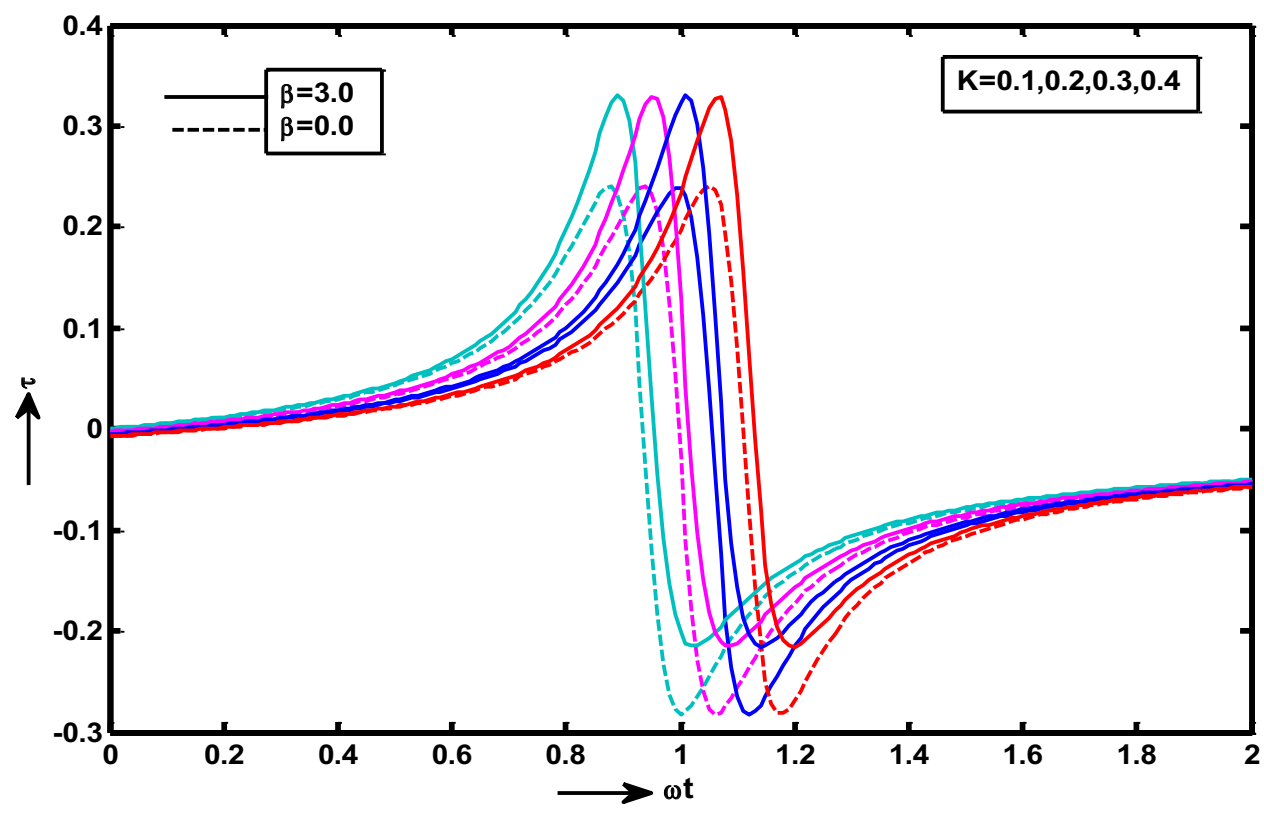

Fig. 11 Influence of $M$ on skin friction coefficient

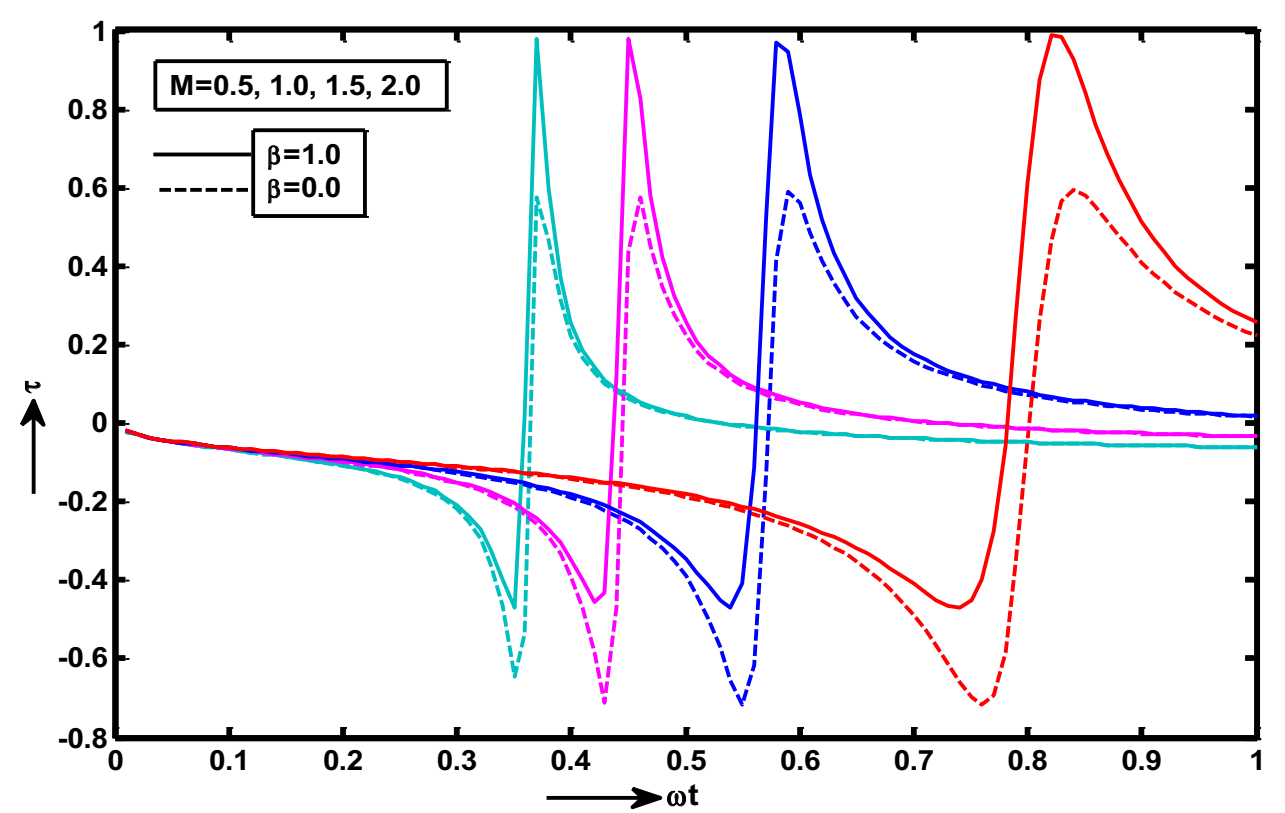

cases $(\beta=0$ and $\beta \neq 0)$. It is clear that the skin friction coefficient vary periodically at asymmetric surface. The variations of heat and mass transfer rates against the time series for different values of $R$ and $K r$ are respectively plotted in Figs. 12 and 13. From these graphs it is clear that $\mathrm{Nu}$ and $S h$ both vary periodically due to asymmetric surface motion.
Figure 14 illustrates the rate of heat transfer against $H$ for dissimilar values of $R$ at the walls $y=h_{1}$ and $y=h_{2}$. It is observed that the growth in $R$ leads to a rise in the values of $\mathrm{Nu}$ at $\mathrm{y}=\mathrm{h}_{1}$ and opposite behaviour at $\mathrm{y}=\mathrm{h}_{2}$. Thus, the rate of heat transfer from the porous asymmetric channel decreases with the increase of $R$. 
Fig. 12 Influence of $R$ on Nusselt number

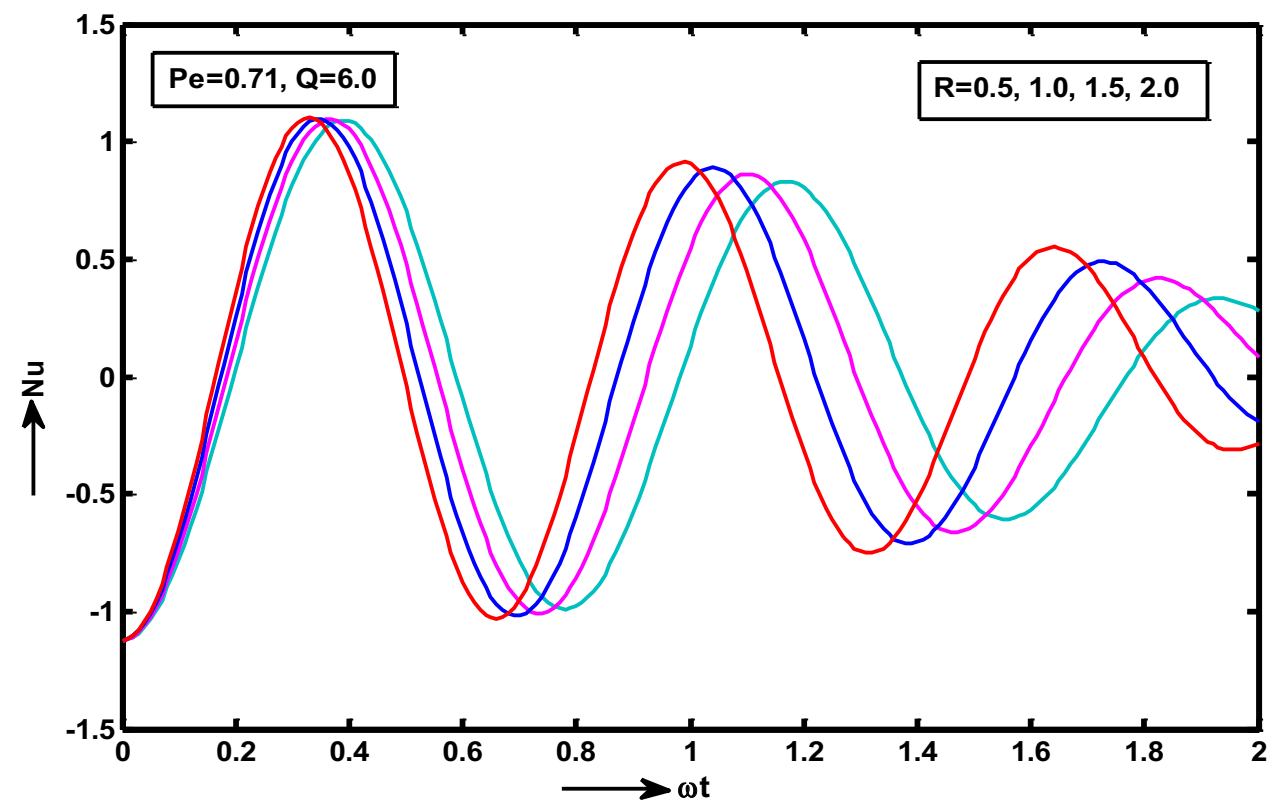

Fig. 13 Influence of $K r$ on Sherwood number

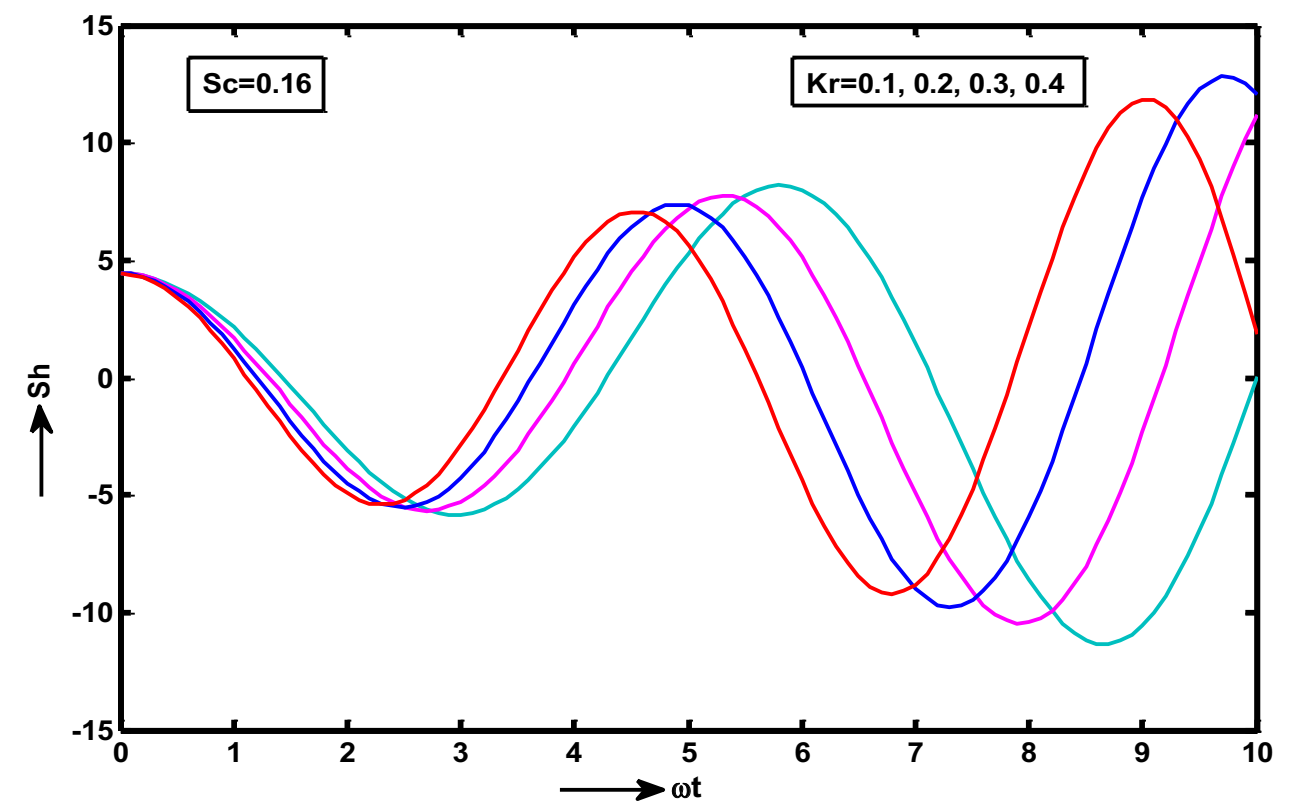

Figure 15 displays the variations of $S h$ against $K r$ for different values of $S c$. It is clear that the rate of mass transfer decline with increase of $S c$ at the wall $y=h_{1}$ whereas the opposite effect at $y=h_{2}$.

\section{Conclusion}

The present study describes the effect of non-Newtonian visco-elastic fluid behaviour of MHD oscillatory flow in an irregular channel. The main conclusions of this study are: 
Fig. 14 Influence of R on Nusselt number

Fig. 15 Influence of Sc on Sherwood number
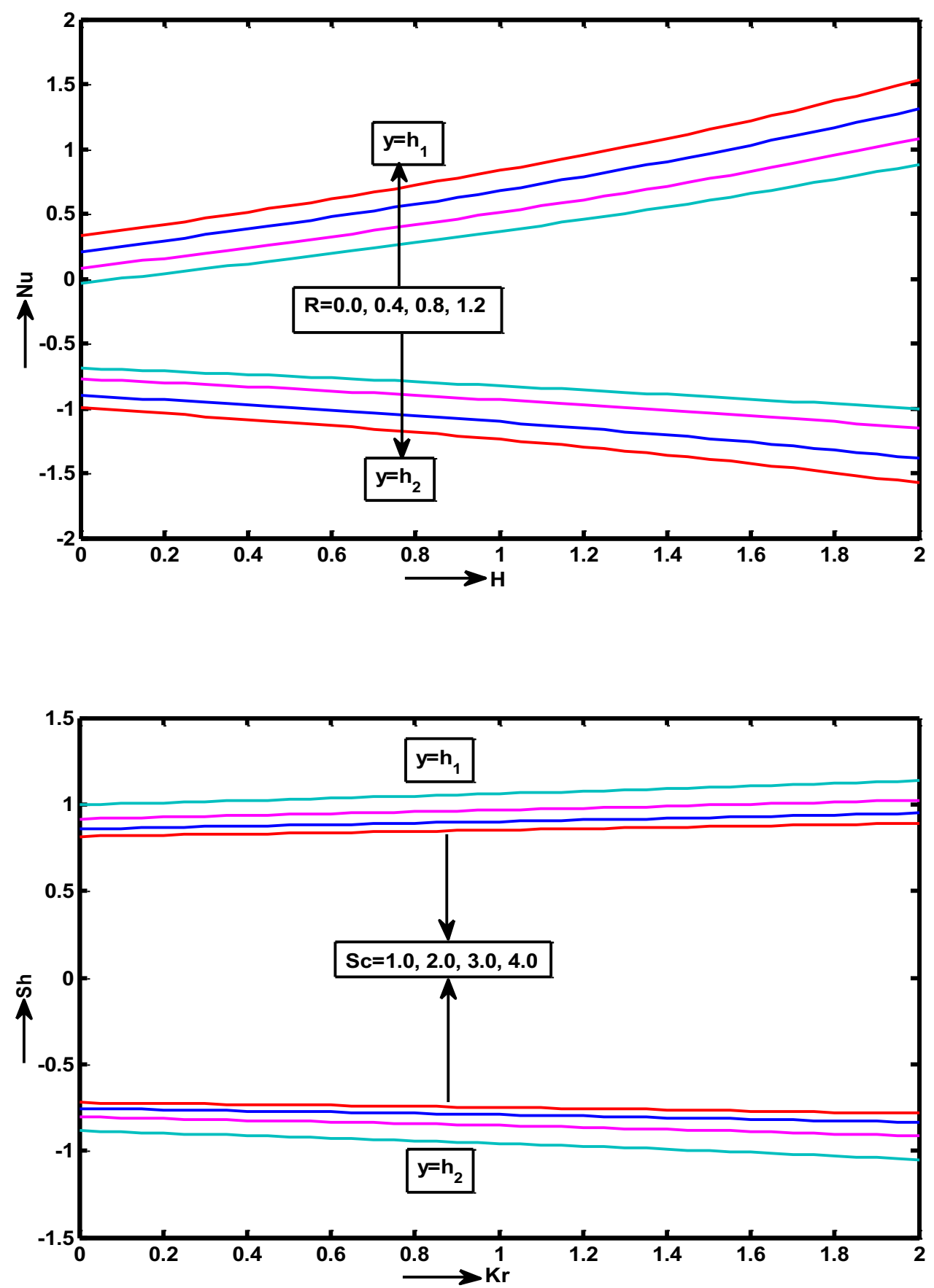

- The temperature profiles increase for all increasing values of $R, Q$ and $P e$ in the fluid flow along an asymmetric wavy channel.

- The velocity and skin friction coefficient in case of Newtonian fluid is more than that of non-Newtonian fluid. This is due to the presence of high viscosity.
- The skin friction coefficient, $\mathrm{Nu}$ and Sh are varying periodically due to surface motion.

- The heat transfer near the wall decrease with the rise of $R$.

- The Sherwood number reduces with rise of $S c$ at the walls $y=h_{1}$ and $y=h_{2}$. 
Authors' contribution All authors contributed equally to the writing of this paper. All authors read and approved the final manuscript.

\section{Compliance with ethical standards}

Conflict of interest The authors declare that they have no competing interests.

Research involving human participants and/or animals This article does not contain any studies with human participants or animals performed by any of the authors.

\section{References}

1. Venkateswarlu B, Satya Narayana PV (2015) MHD visco-elastic fluid flow over a continuously moving vertical surface with chemical reaction. Walailak J Sci Technol 12(9):1-9

2. Satya Narayana PV, Venkateswarlu B, Devika B (2016) Chemical reaction and heat source effects on MHD oscillatory flow in an irregular channel. Ain Shams Eng J 7:1079-1088

3. Misra JC, Mallick B, Sinha A (2016) Heat and mass transfer in asymmetric channels during peristaltic transport of an MHD fluid having temperature-dependent properties. Alex Eng J 57(1):391-406

4. Ramesh K (2016) Influence of heat and mass transfer on peristaltic fluid of a couple stress fluid through porous medium in the presence of inclined magnetic field in an inclined asymmetric channel. J Mol Liq 219:256-271

5. Ramesh K, Devakar M (2017) Effect of heat transfer on the peristaltic transport of a MHD second grade fluid through a porous medium in an inclined asymmetric channel. Chin J Phys 55(3):825-844

6. Shamsi MR, Akbari OA, Marzban A, Toghraie D, Mashayekhi $R$ (2017) Increasing heat transfer of non-Newtonian nanofluid in rectangular microchannel with triangular ribs. Phys $E$ 93:167-178

7. Eldabe NT, Abou-zeid MY (2017) Homotopy perturbation method for MHD pulsatile non-Newtonian nanofluid flow with heat transfer through a non-Darcy porous medium. J Egypt Math Soc 25(4):375-381

8. Kefayati GHR, Tang H (2017) Simulation of natural convection and entropy generation of MHD non-Newtonian nanofluid in a cavity using Buongiorno's mathematical model. Int J Hydrog Energy 42(27):17284-17327

9. Khan Z, Shah RA, Altaf M, Islam S, Khan A (2018) Effect of thermal radiation and MHD on non-Newtonian third grade fluid in wire coating analysis with temperature dependent viscosity. Alex Eng J 57(3):2101-2112

10. Abdul Hakeem AK, Saranya S, Ganga B (2017) Comparative study on Newtonian/non-Newtonian base fluids with magnetic/nonmagnetic nanoparticles over a flat plate with uniform heat flux. J Mol Liq 230:445-452

11. Thurston GB (1979) Rheological parameters for the viscosity, viscoelasticity and thixotropy of blood. Biorheol 16:149-162

12. Thurston GB (1972) Viscoelasticity of human blood. Biophys J 12:1205-1217

13. Misra JC, Paul B, Gupta AS (1998) Hydromagnetic flow of a second-grade fluid in a channel-some applications to the physiological systems. Math Models Methods Appl Sci 8(8):1323-1342

14. Misra JC, Maiti S, Shit GC (2008) Peristaltic transport of a physiological fluid in an asymmetric porous channel in the presence of an external magnetic field. J Mech Med Biol 8(4):507-525
15. Misra JC, Shit GC, Chandra S, Kundu PK (2011) Electroosmotic flow of a viscoelastic fluid in a channel: applications to physiological fluid mechanics. Appl Math Comput 217(20):7932-7939

16. Tripathi D, Anwar Beg O (2012) A study of unsteady physiological magneto-fluid flow and heat transfer through a finite length channel by peristaltic pumping. J Eng Med 226(8):631-644

17. Hayat T, Yasmin H, Al-Yami M (2014) Soret and Dufour effects in peristaltic transport of physiological fluids with chemical reaction: a mathematical analysis. Comput Fluids 89:242-253

18. Misra JC, Adhikary SD (2016) MHD oscillatory channel flow, heat and mass transfer in a physiological fluid in presence of chemical reaction. Alex Eng J 55(1):287-297

19. Ponalagusamy $R$ (2018) Mathematical analysis of flow of non-Newtonian fluid due to metachronal beating of cilia in a tube and its physiological applications. Appl Math Comput 337:545-561

20. Nlkiforov VN (2007) Magnetic induction hyperthermia. Russ Phys J 50(9):913-924

21. Jordan A, Wust P, Scholz R, Tesche B, Fahling H, Mitrovics $T$, Vogl T, Carvos-Navarro J, Felix R (1996) Cellular uptake of magnetic fluid particles and their effects on human adenocarcinoma cells exposed to AC magnetic fields in vitro. Int J Hyperth 12(6):705-722

22. Raftari Behrouz, Vajravelu Kuppalapalle (2012) Homotopy analysis method for MHD viscoelastic fluid flow and heat transfer in a channel with a stretching wall. Commun Nonlinear Sci Numer Simul 17(11):4149-4416

23. Singh KD (2013) Effect of slip condition on viscoelastic MHD oscillatory forced convection flow in a vertical channel with heat radiation. Int J Appl Mech Eng 18(4):1237-1248

24. Singh KD (2013) MHD mixed convection visco-elastic slip flow through a porous medium in a vertical porous channel with thermal radiation. Kragujev J Sci 35:27-40

25. Abd-Alla AM, Abo-Dahab SM (2015) Magnetic field and rotation effects on peristaltic transport of a Jeffrey fluid in an asymmetric channel. J Magn Magn Mater 374:680-689

26. Makinde OD, Mhone PY (2015) Heat transfer to MHD oscillatory flow in a channel filled with porous medium. Rom J Phys 20:931-938

27. Ghosh SK (2007) Hydromagnetic fluctuating flow of a viscoelastic fluid in a porous channel. J Appl Mech 74(2):177-180

28. Mehmood A, Ali A (2007) Effect of slip condition on unsteady MHD Oscillatory flow of a viscoelastic fluid in a planar channel. Rom J Phys 52:85-91

29. Ali F, Khan I, Mustapha N, Shafie S (2012) Unsteady MHD oscillatory flow of viscoelastic fluids in a porous channel with heat and mass transfer. J Phys Soc Jpn 81(6):064402

30. Devika B, Satya Narayana PV, Venkataramana S (2013) MHD oscillatory flow of a Visco elastic fluid in a porous channel with chemical reaction. Int J Eng Sci Invent 2:2319-2672

31. Gireesha BJ, Mahanthesh B (2013) Perturbation solution for radiating viscoelastic fluid flow and heat transfer with convective boundary condition in non uniform channel with Hall current and chemical reaction. Thermodynamics 1-14

32. Nayak MK, Dash GC, Singh LP (2014) Effect of chemical reaction on MHD flow of a visco-elastic fluid through porous medium. $J$ Appl Anal Comput 4(4):367-381

33. Venkateswarlu B, Satya Narayana PV, Devika B (2017) Effects of chemical reaction and heat source on MHD oscillatory flow with heat and mass transfer of a viscoelastic fluid in a vertical channel with porous medium. Int J Appl Comput Math 3:937-952

34. Muthuraj R, Srinivas $S$ (2010) A note on heat transfer to MHD oscillatory flow in an asymmetric wavy channel. Int Commun Heat Mass Transf 37:1255-1260 
35. Ogulu A, Bestman AR (1993) Deep heat muscle treatment-a mathematical model-I. Acta Phys Hung 73:3-16

36. Ogulu A, Abbey TM (2005) Simulation heat transfer on an oscillatory blood flow in an indented porous artery. Int Commun Heat Mass Transf 32:983-989

37. Makinde OD (2007) Asymptotic approximations for oscillatory flow in a tube of varying cross-section with permeable isothermal wall. Rom J Phys 52(1-2):59-72

38. Mishra SR, Dash GC, Acharya M (2013) Mass and heat transfer effect on MHD flow of a visco-elastic fluid through porous medium with oscillatory suction and heat source. Int J Heat Mass Transf 57(2):433-438
39. Oahimire Jl, Olajuwon BI (2014) Effect of Hall current and thermal radiation on heat and mass transfer of a chemically reacting MHD flow of a micropolar fluid through a porous medium. $J$ King Saud Univ Eng Sci 26:112-121

Publisher's Note Springer Nature remains neutral with regard to jurisdictional claims in published maps and institutional affiliations. 